313 OCCUPATION-SPECIFIC FREQUENCY OF SICK-LEAVE DUE TO VARICOSE VEINS OF THE LOWER EXTREMITIES

U Latza, Brendler, Liebers. Federal Institute for Occupational Safety and Health, Berlin, Germany

10.1136/oemed-2013-101717.313

Objectives Chronic venous disorders of the lower limbs are a frequent condition in the general population. In the working population, prolonged standing at work is associated with the development of diseases of varicose veins. The aim is to identify high risk occupations based on data on sick-leave due to varicose veins of the lower extremities.

Methods The analysis was based on a secondary data analysis of sick-leave of almost all German statutory health insurances in 2008. The database contains aggregated data of approximately 26.2 million insured employees. The occurrence of sickleave events due to the diagnosis varicose veins of the lower extremities (ICD-10 I83) is defined as outcome variable. The assignment of employees to occupational groups (according to Blossfeld 1985 based on the German Classification of Occupations) is considered as exposure variable. Morbidity ratios (SMR) stratified by sex and age group with $99.9 \%$ confidence intervals were estimated (reference: qualified mercantile and administrative occupations).

Results Compared to the reference group the expected number of sick-leave was particularly increased for manual workers with low skill level (men: SMR 1.61 [1.55-1.68], women: SMR 1.51 [1.43-1.58]) and high skill level (men: SMR 1.61 [1.55-1.68], women SMR 1.73 [1.61-1.84]). In both genders, sick-leave was also higher than expected for low-skilled mercantile and administrative occupations as well as low-skilled service workers. An example of a high skilled manual occupation with an increased risk is confectioner (men: SMR 3.08 [1.92-4.63], women: SMR 2.74 [1.78-4.00]). Among women, the risk of semi-professions, qualified service workers, and agricultural workers was also higher than the reference group.

Conclusions An ageing workforce is expected in the future. Thus, interventions focussed on the reduction of prolonged standing particularly among manual occupations in production and service should be initiated and evaluated for the primary and secondary prevention of varicose veins.

\section{TRANSGENERATIONAL SICKNESS ABSENCE PATTERNS IN NORWEGIAN FAMILIES}

${ }^{1} \mathrm{P}$ K Kristensen, ${ }^{1}$ Mehlum, ${ }^{1}$ Corbett, ${ }^{2}$ Bjerkedal. ' National Institute of Occupational Health, Oslo, Norway; ${ }^{2}$ University of Oslo, Oslo, Norway

\subsection{6/oemed-2013-101717.314}

Objectives Sickness absence (SA) rates are high in Norway, and there is an increasing gender disparity with a female excess. Social interaction on different levels has been suggested as one potential determinant of SA. Our objective was to examine the effect of maternal SA in adolescence on own SA in young adult age. The hypotheses were that maternal SA has an impact on own subsequent SA, with females being more susceptible than males.

Methods All live-born in Norway 1974-1976 ( $\mathrm{N}=169$ 498) were followed up in several national registers. The analyses included the 130645 subjects at risk of sickness absence in 2003 who had a mother at risk of SA in 1990. All SA was restricted to spells exceeding 16 days. The exposure was any maternal SA in 1990. The outcome was any non-injury musculoskeletal (MSD) SA (ICPC codes L72-L81, L96) in 2003. Gender-specific one-year MSD SA risk was computed, and associations with maternal SA were examined as relative risks (RR) in Poisson regression. Estimates were adjusted for predetermined covariates (year of birth, birth order, parental education level and marital status, maternal age, geographical region).

Results MSD SA risks were 0.070 (4381/62 221) for females and 0.039 (2638/68 424) for males. Crude RRs in association with maternal SA was 1.31 (95\% CI 1.20-1.42) for females and 1.45 (1.31-1.61) for males. Adjusted RRs were 1.27 (1.19-1.36) and 1.35 (1.21-1.49), respectively. On an additive scale, adjusted risk differences were $0.017(0.010-0.023)$ for females and 0.013 (0.009-0.018) for males.

Conclusions Maternal SA was moderately associated with own MSD SA 14 years later. The results suggest that social interaction within the family has some impact on SA in young adult age. There were no indications that females were more susceptible than males concerning this maternal influence.

\section{TIME TO RETURN TO WORK FROM SICKNESS ABSENCE IN HEALTH CARE WORKERS}

${ }^{1} \mathrm{C}$ S Serra, ${ }^{2}$ Vargas-Prada, ${ }^{3}$ Mirabent, ${ }^{3}$ Villar, ${ }^{3}$ Ramada, ${ }^{4}$ Delclos, ${ }^{2}$ Benavides. ${ }^{1}$ Barcelona, Spain; ${ }^{2}$ Center for Research in Occupational Health (CISAL). Universitat Pompeu Fabra, Barcelona, Spain; ${ }^{3}$ Parc de Salut Mar, Barcelona, Spain; ${ }^{4}$ University of Texas School of Public Health, Houston, United States of America

\subsection{6/oemed-2013-101717.315}

Objectives A comprehensive programme to facilitate return to work (RTW) was started in 2011 in a health care centre with a sustained impact until now. The objective of this study was to analyse time to RTW to guide interventions in more specific groups. Methods The study population was a cohort of 5,881 health care workers in a large health institution during 2011 that includes two acute care centres, a psychiatry institute, one long term care centre, two schools of health studies and one research centre. Data on the study population and sickness absences (SA) were obtained through electronic records of Human Resources Department. Incidence of SA per 100 workers was described. Median SA durations and interquartile ranges were calculated using Wang-Chang estimator. Multilevel poisson-based conditional fragility models were used to calculate time to RTW hazard ratios (HRs) and associated 95\% confidence intervals (CI95\%). A HR lower than 1 expresses a longer duration of SA until RTW than the reference group.

Results A total 787 episodes of SA were identified, of which 608 (77\%) lasted more than 7 days. Incidence of SA was higher in women, nurses' aides and nurses, those with a permanent contract, in direct patient care, psychiatry and geriatrics, and increased with age. Differences within groups tended to be higher for long ( $>7$ days) than short episodes. Regarding time to RTW, adjusted HRs (HRa) decreased for older workers (HRa 4554years: 0.37; CI95\% 0.19-0.37; HRa 55-64years: 0.27; CI95\% $0.13-0.53)$, and were associated with holding a permanent contract (HRa 0.60; CI95\% 0.39-0.91). Non statistically significant associations were found for sex, centre, occupation or type of care.

Conclusions Ageing population at work plays an important role and interventions to facilitate RTW are essential to maintain people active. Presenteeism or working even when having a health condition, should be seriously addressed. 